\title{
Considerazioni su un caso di actinomicosi cervicale
}

\author{
Amelia Romano', Salvatore Giordano', Laura Infurnari', Giuseppe Lipani², \\ Lorenzo Marasà', Francesco Scarlata' \\ 'Istituto di Patologia Infettiva e Virologia dell'Università di Palermo \\ ${ }^{2}$ UO Anatomia Patologica - PO Ascoli - ARNAS Civico - Ascoli - Di Cristina - Palermo
}

Key words: Lymphoadenopaty, actinomycosis, flogosys

\section{Remarks on a case of cervical actinomycosis}

\section{SUMMARY}

Actinomycosis is a bacterial infection due to Gram positive bacteria of Actinomyces genus. Actinomyces israelii is the most common in human infection. The authors described a case occurred in a 43 aged woman with a clinical presentation of a right submandibular indurative mass. After surgery, histological examination showed a granulomatous tissue with many actinomycetes.

Patient was submitted to treatment with i.v penicillin. On the $\mathrm{I}^{\text {th }}$ day antibiotic therapy was replaced with ceftriaxone and teicoplanina. Patient did not present intercurrence during this period and presented improvement of the lesion.Actinomycosis is an uncommon disease. Establishment of the definite diagnosis requires a high index of suspicion in all cases of relapsing cervical lesions. Penicillin is effective and it can be replaced by third-generation cephalosporins.

L'actinomicosi è un'infezione batterica endogena, ad andamento lentamente progressivo, causata da batteri Gram positivi appartenenti al genere Actinomyces, tra i quali Actinomyces israelii è quello più frequentemente causa di infezione umana.

Si tratta di un commensale abituale del cavo orale umano che, occasionalmente, in talune circostanze locali favorenti, specie se in grado di ridurre il potenziale di ossido riduzione, può invadere i tessuti profondi provocando un'infiammazione granulomatosa cronica seguita da necrosi e fibrosi, capace di diffondersi alle strutture contigue e di formare fistole e cavità (2).

Il 50\% dei casi di actinomicosi si osserva in sede cervico-faciale (1). Probabili fattori predisponenti sarebbero stati individuati nella scarsa igiene orale, formazione di soluzioni di continuo della barriera mucosa orale, estrazioni dentarie, traumi, e infezioni batteriche dell'orofaringe (7).

\section{CASO CLINICO}

C.M.G., donna di 43 anni, residente a Palermo. Sei mesi prima di arrivare alla nostra osservazione, tumefazione in sede sottomandibolare destra non dolente e non accompagnata da febbre. Due mesi dopo la comparsa di tale tumefazione veniva sottoposta a resezione chirurgica della ghiandola sottomandibolare destra.

L'esame istologico del suddetto resecato chirurgico, delle dimensioni di $\mathrm{cm} 4 \times 2 \times 1,5$, escludeva la presenza di patologia a carico della ghiandola salivare, bensì evidenziava nel tessuto adiacente la ghiandola un processo granulomatoso ascessualizzante nel cui contesto si riscontrava la presenza di granulociti neutrofili frammisti ad ammassi di filamenti miceliali con rigonfiamento terminale a guisa di "clava" da riferire a colonie actinomicotiche

(figura I). La semina di materiale bioptico su terreno Agar sangue, in atmosfera anaerobica e alla temperatura di $37^{\circ} \mathrm{C}$, portava dopo circa una settimana allo sviluppo di tipiche colonie "a dente molare" rilevate, a cumulo, bianche, lucenti, con margini irregolari riferibili ad Actinomyces spp. Successivamente, dopo circa quattro mesi, la paziente veniva sottoposta ad esame ecografico di controllo in sede sottomandibolare destra con il quale era possibile osservare multiple aree anecogene di tipo cistico di dimensioni inferiori al centimetro da riferire ad aree microascessuali accompagnate da linfonodi reattivi giugulari e laterocervicali destri. Solo allora la paziente arrivava alla nostra osservazione.

Oltre agli esami ematochimici di routine, veniva sottoposta ad intradermoreazione di Mantoux risultata positiva $(\varnothing 5 \mathrm{~cm})$ e, quindi, successivamente ad esame radiografico del torace con il quale si apprezzava una piccola calcificazione centimetrica in sede paramediastinica superiore destra. Per tale motivo veniva eseguita ricerca del Bacillo di Koch tramite metodica PCR (Cobas Amplicor M. tuberculosis - Roche) su tre campioni di urine e su tampone cutaneo del materiale purulento fuoriuscito dalla fistola.

La ricerca è risultata negativa in entrambi i casi. 
Pertanto, in base alle evidenze cliniche, istologiche, microbiologiche ed ecografiche, si decideva di intraprendere una terapia antibiotica appropriata al caso con Penicillina G (5.000.000 UI x 4 e.v.). In undicesima giornata la Penicillina veniva sostituita con Ceftriaxone alla dose di $1 \mathrm{~g}$ x 2 e.v. per due giorni, e successivamente, alla dose di $1 \mathrm{~g} /$ die i.m. per altri sette giorni.

In seguito al riscontro in corrispondenza della cicatrice chirurgica di un'area eritematosa, dolente e calda al termotatto con fistola esterna da cui fuoriusciva materiale purulento, si decideva di aggiungere allo schema terapeutico Teicoplanina $200 \mathrm{mg} /$ die.

La risposta alla nostra terapia (peraltro priva di effetti indesiderati) è risultata soddisfacente e i controlli a distanza hanno evidenziato la chiusura della fistola ed hanno escluso recidive locali.

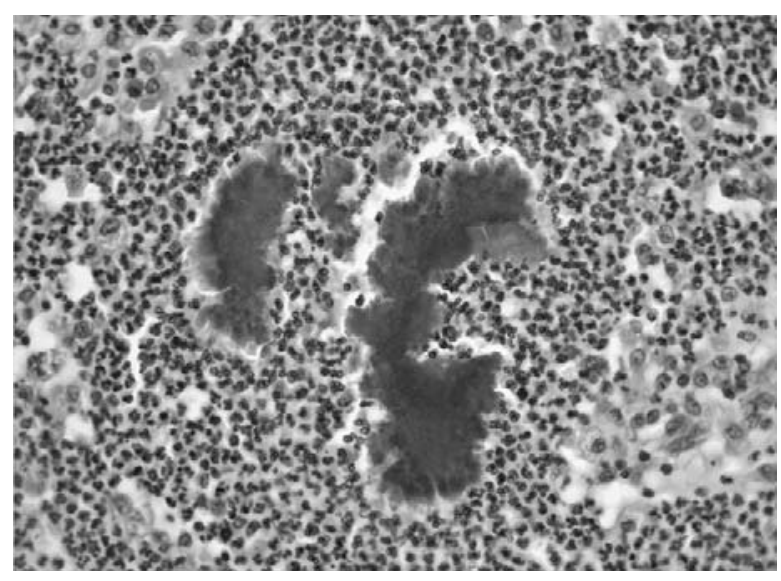

Figura I. Esame istologico del resecato chirurgico: processo granulomatoso ascessualizzante nel cui contesto si evidenziano colonie riferibili ad actinomiceti.

\section{DISCUSSIONE}

L'actinomicosi è oggi un'infezione molto rara e rarissime sono le segnalazioni riportate sulla letteratura internazionale. Una migliore igiene dentale e l'uso tempestivo degli antibiotici nei processi infettivi orofaringei hanno, infatti, contribuito a ridurre notevolmente l'incidenza di questa malattia. Ciò ha determinato tuttavia una minore dimestichezza con tale patologia e troppo spesso la diagnosi viene posta soltanto in seguito ad un intervento chirurgico demolitivo tramite esame istologico o in fase avanzata.

Considerato che l'actinomicosi può manifestarsi in molteplici forme e che l'unica caratteristica fondamentale è la presenza di focolai purulenti circondati da fibrosi compatta, è necessario prendere sempre in considerazione la sua diagnosi in caso di lesioni o infezioni recidivanti di testa $o$ collo (6).
La diagnosi differenziale con un processo neoplastico o con una infezione tubercolare può risultare spesso difficoltosa, ma il riscontro microscopico dei caratteristici grani gialli actinomicotici nel pus o nel materiale di drenaggio è altamente indicativo di actinomicosi e risolve ogni dubbio diagnostico evitando di utilizzare metodiche invasive.

La Penicillina $\mathrm{G}$ ha dimostrato ancora una volta di essere il farmaco di elezione per l'infezione da actinomiceti, seguita con ottimi risultati dalla somministrazione di Ceftriaxone $(4,5)$.

È consigliabile inoltre associare allo schema terapeutico un ulteriore antibiotico attivo su germi Gram positivi, Teicoplanina nel nostro caso, in considerazione del fatto che la maggiorparte delle infezioni da actinomiceti è polimicrobica (Enterobatteri, Stafilococchi e Streptococchi sono stati spesso isolati insieme agli actinomiceti) (3).

\section{BIBLIOGRAFIA}

1. Bennhaff D. Actinomycosis: Diagnostic and therapeutic considerations and a review of 32 cases. Laryngoscope 1984; 94: 1198-217.

2. Brown J. Human actinomycosis: A study of 181 subjects. Hum Pathol 1973; 4: 319-30.

3. Holm P. Studies on aetiology of human actinimycosis.I. The "other" microbes of actinomycosis and their importance. Acta Pathol Microbiol Scand 1950; 27: 736.

4. Peabody J, Seabury J. Actinomycosis and nocardiosis: A review of basic differences in therapy. Am J Med 1960; 60: 99-115.

5. Skoutelis A, Petrochilos J, Bassoris H. Successful treatment of thoracic actinomycosis with ceftriaxone. Clin Infect Dis 1994; 19: 161-2.

6. Spilsbury BW, Johnstone FRC. The clinical course of actinomycotic infections: a report of 14 cases. Can J surg 1962; 5: 33-48.

7. Zitsch RP $3^{\text {rd }}$, Bothwell M. Actinomycosis: A potential complication of head and neck surgery. Am J Otolaryngol 1999; 20: 260-2.

\section{Salvatore Giordano}

Istituto di Patologia Infettiva e Virologia

Via Benedettini 1 - 90144 Palermo

Fax 0916514634 - Tel 0916666276

E-mail: giordano.s@tiscalinet.it 